\title{
RESISTANCE TO VITAMIN D TREATMENT AS AN INDICATION OF CELIAC DISEASE IN A PATIENT WITH PRIMARY HYPOPARATHYROIDISM
}

\author{
doi: $10.1590 / \mathbf{S 1 8 0 7 - 5 9 3 2 2 0 0 9 0 0 0 3 0 0 0 1 8}$
}

José Antonio Miguel Marcondes, Pedro Seferian Junior, Cristina Aparecida Proques da Silveira Mitteldorf

\section{INTRODUCTION}

Hypocalcemia may present as an asymptomatic laboratory finding or as a severe, life-threatening condition. Upon the diagnosis of acute hypocalcemia, a rapid treatment regimen may be necessary. In contrast, chronic hypocalcemia may be well tolerated by the patient, but treatment nonetheless remains necessary in order to prevent long-term complications.

The hallmark of acute hypocalcemia is neuromuscular irritability, although paresthesias of the extremities may also occur, along with fatigue and anxiety. In addition, very painful muscle cramps may develop and can progress into carpal spasms or tetany. ${ }^{1}$ From a clinical perspective, neuromuscular irritability can be demonstrated by testing for Chevostek or Trousseau signs. However, all of these symptoms can be corrected by the administration of calcium replacement treatments.

The observation of an inappropriately normal PTH coupled with hypocalcemia should promptly warrant the diagnosis of hypoparathyroidism, which may be transient, genetically inherited, or acquired due to an autoimmune process. It may also follow surgery or neck irradiation treatment. ${ }^{1}$

In this paper, we present a case study of a patient with primary hypoparathyroidism who is surprisingly resistant to the usual treatment consisting of calcium and vitamin D supplementation. The coincident observation of chronic diarrhea prompted us to consider the possibility of a malabsorption syndrome with steatorrhea, since vitamin D absorption is fat-dependent.

\section{CASE REPORT}

A 39-year-old woman was admitted to the emergency

Hospital Sírio Libanês - São Paulo/SP, Brazil.

Email: marcondesmd@uol.com.br room at our facility with tetany that was resolved by the intravenous administration of calcium gluconate in high doses. The patient's recent medical history revealed recurrent episodes of diarrhea, asthenia, paresthesias and weight loss $(7.0 \mathrm{~kg})$ over a one-month period. Approximately one year prior to this emergency room admission, the patient underwent a quadrantectomy for breast cancer. Overall, the patient's family history was unremarkable. At the time of admission, the patient was being treated with tamoxifen.

Subsequent physical examination revealed a lean woman (body mass index of $22.7 \mathrm{~kg} / \mathrm{m}^{2}$ ) with a blood pressure of 120 over $60 \mathrm{mmHg}$ and a heart rate of 100 beats per minute. In addition, the patient had a $+/ 3$ bilateral maleolar edema and no vitiligo was observed. The thyroid and the abdomen of the patient appeared normal.

Initial laboratory analyses revealed that the patient was hypocalcemic (total calcium of $5.4 \mathrm{mg} / \mathrm{dL}$ and ionized calcium of $0.74 \mathrm{mM} / \mathrm{L})$, hypoalbuminemic $(2.4 \mathrm{~g} / \mathrm{dL})$ and presented microcytic hypochromic anemia $(9.4 \mathrm{~g} / \mathrm{dL})$. The PTH (30 nM/L), phosphorus $(4.4 \mathrm{mg} / \mathrm{dL})$ and magnesium concentrations $(2.1 \mathrm{mg} / \mathrm{dL})$ were normal. A liver functional test revealed a high prothrombin time ( 25 seconds) with normal transaminases and bilirubin levels. In addition, renal and thyroid function tests were normal.

The patient was placed on a high protein diet and treated with oral calcium gluconate and calcitriol with a stepwise increase in the dosage of up to $1.0 \mathrm{mg} /$ day, with persistence of the paresthesia, Chevostek and Trousseau signs, and maleolar edema and without significant changes in metabolic and nutritional parameters (Figure 1). The patient's serum analysis indicated the presence of immunoglobulin A isotype antibodies against endomysium (1/640). Diagnosis of gluten-sensitive enteropathy or celiac disease was suggested at endoscopy, due to flat mucosa, and confirmed by histological findings in duodenal biopsy, including absent villi, crypt hyperplasia and T-cell lymphocytic infiltration in the epithelium. Eleven days after admitting the patient, she was placed on a gluten-free diet, which rapidly lead 

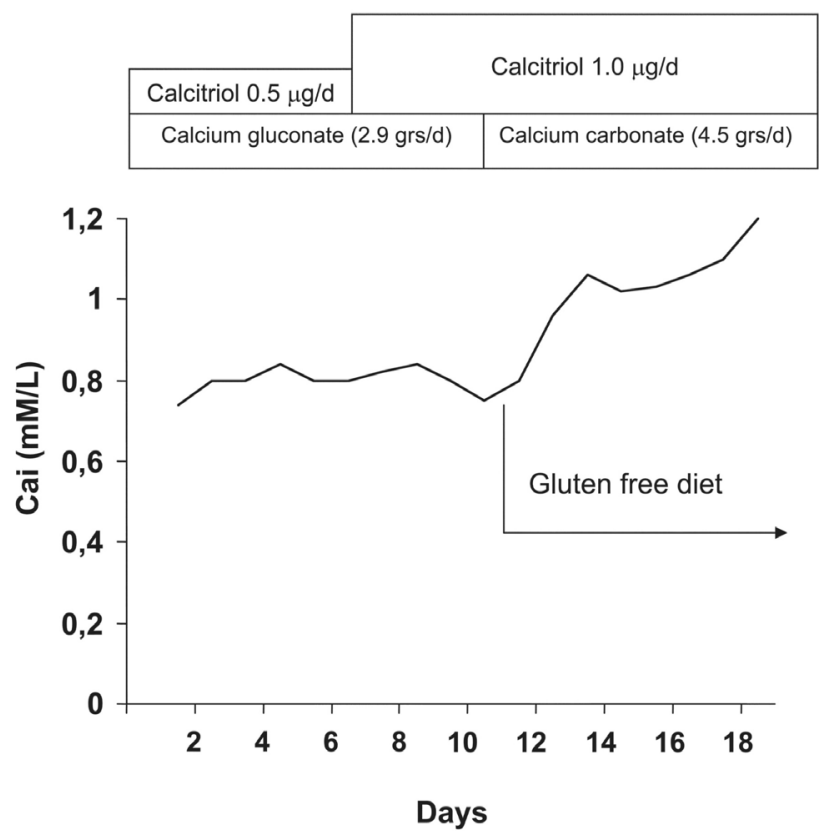

Figure 1 - The effect of a gluten-free diet on the ionized calcium levels in a patient with primary hypoparathyroidism associated with celiac disease

to the normalization of her bowel habits and improved her metabolic and nutritional parameters.

The patient was discharged from the hospital after 7 days on a gluten-free diet supplemented with daily doses of calcitriol (1.0 mg/day) and calcium carbonate (4.5 g/day). Her body mass index was $25.5 \mathrm{~kg} / \mathrm{m}^{2}$ and blood pressure and heart rate were 120 over $80 \mathrm{mmHg}$ and 80 bets per minute, respectively. Maleolar edema was no longer observed and the Chevostek and Trousseau signs were negative. In addition, the levels of total calcium, ionized calcium, albumin and hemoglobin increased to $8.3 \mathrm{mg} / \mathrm{dL}, 1.1 \mathrm{mM} / \mathrm{L}, 4.2 \mathrm{~g} / \mathrm{dL}$ and $11.3 \mathrm{~g} / \mathrm{dL}$, respectively, while the prothrombin time decreased from 25 seconds to 11 seconds.

Six months thereafter, although the patient still reported occasional episodes of diarrhea, assays for the endomysial antibody were negative.

\section{DISCUSSION}

This patient had a history of chronic diarrhea and weight loss. Upon admission, laboratory analyses indicated that the patient had low levels of total calcium and ionized calcium that were initially observed under conditions of inappropriately normal levels of PTH and magnesium. We diagnosed the patient with primary hypoparathyroidism and she was treated with intravenous calcium gluconate and vitamin $\mathrm{D}_{3}$ (calciferol) supplements that were administered via a stepwise increase of up to $0.25 \mathrm{mg}$ four times a day. This is considered the maximum daily recommended dose.
Because the hypocalcemia persisted in the patient, we considered changing the diagnosis to malabsorption syndrome due to the presence of chronic diarrhea, since vitamin D absorption is fat-dependent. Assays for the presence of the endomysial antibody were positive and a subsequent biopsy of duodenal mucosa revealed results that were consistent with gluten-sensitive enteropathy or celiac disease. We then administered the patient a gluten-free diet in combination with vitamin D supplementation. This was followed by an encouraging increase in the levels of serum calcium over a period of several days until the patient's bowel habits normalized and the symptoms of hypocalcemia reappeared.

Celiac disease is an immune disorder triggered by an environmental agent (the gliadin component of gluten) in genetically predisposed individuals. ${ }^{3}$ Epidemiological studies using $\operatorname{Ig}$ A antibodies to gliadin and endomysium had a positive predictive value of $100 \%$, with a prevalence of 1:22 in first degree relatives of patients with celiac disease and a prevalence of 1:133 in the not-at-risk groups. ${ }^{4}$

Hypocalcemia in celiac disease may arise from the impaired absorption of vitamin D or the loss of calcium through the binding of intraluminal calcium to unabsorbed fatty acids. Both secondary hypoparathyroidism due to hypomagnesemia ${ }^{5}$ and compensatory secondary hyperparathyroidism have been described elsewhere. ${ }^{6}$

On the other hand, only a few reports have been published on the coexistence of primary hypoparathyroidism and celiac disease. ${ }^{7-14}$ The age of the disease onset ranged from early childhood ${ }^{14}$ to old age. ${ }^{12}$ In some reports, such as one describing a 62-year-old woman, hypoparathyroidism was diagnosed 33 years prior to the diagnosis of celiac disease, ${ }^{12}$ while in other reports, such as a report characterizing a 60 year-old man, ${ }^{7}$ the diagnosis of hypoparathyroidism was made concomitantly with the diagnosis of celiac disease. In a separate report, a patient with idiopathic autoimmune hypoparathyroidism who developed autoimmune hyperthyroidism (Graves' disease) was subsequently diagnosed with celiac disease. ${ }^{13}$ The malabsorption of $l$-thyroxine was the only indication of the presence of celiac disease. In fact, a common immunological basis that associated the idiopathic hypothyroidism with the celiac disease was hypothesized in this instance. ${ }^{9}$ In yet another report, celiac disease was diagnosed in a patient with hypoparathyroidism in the absence of gastrointestinal symptoms. ${ }^{11}$

In conclusion, the possibility of celiac disease should be considered in patients with hypoparathyroidism that seems unduly difficult to treat. This should be evaluated even in the absence of gastrointestinal symptoms. 


\section{REFERENCES}

1. Maeda SS, Fortes EM, Oliveira UM, Borba VC, Lazaretti-Castro M. Hypoparathyroidism and pseudohypoparathyroidism. Arq Bras Endocrinol Metabol. 2006;50:664-73.

2. Presutti RJ, Cangemi JR, Cassidy HD, Hill DA. Celiac disease. Am Fam Physician. 2007;76:1795-802.

3. PittschieleR K, Ladinser B. Celiac disease: screened by a new strategy. Acta Paediatr. 1996;412:42-5.

4. Fasano A, Berti I, Gerarduzzi T, Not T, Colletti RB, Drago S et al. Prevalence of celiac disease in at-risk and not-at-risk groups in the United States: a large multicenter study. Arch Intern Med. 2003;163:286-92.

5. Foulston C, Gall G, Mitchell I, Cooper DM, Scott RB. Transient neutral fat steatorrhea, elevated sweat chloride concentration, and hypoparathyroidism in a child with celiac disease. J Pediat Gastroenterol Nut. 1985;4:143-5.

6. Rickels R, Mandel S. Celiac disease manifesting as isolated hypocalcemia. Endocr Pract. 2004;10:203-7.

7. Matsueda K, Rosenberg IH. Malabsorption with idiopathic hypoparathyroidism responding to treatment for coincident celiac sprue. Dig Dis Sci. 1982;27:269-73.
8. Cataldo F, Violante M, Bellia L, Albeggiani A. Clinical remarks on a case of hypocalcemic tetany secondary to celiac disease initially diagnosed as chronic hypoparathyroidism. Minerva Pediatr. 1989;41:113-6.

9. Wortsman J, Kumar V. Case report: idiopathic hypoparathyroidism co-existing with celiac disease: immunologic studies. Am J Med Sci. 1994;307:420-7.

10. Frysák Z, Hrcková Y, Rolinc Z, Hermanová Z, Lukl J. Idiopathic hypoparathyroidism with celiac disease: diagnostic and therapeutic problem. Vnitr Lek. 2000;46:408-12.

11. Sari R, Yildirim B, Sevinc A, Buyukberber S. Idiopathic hypoparathyroidism and celiac disease in two patients with previous history of cataract. Indian J Gastroenterol. 2000;19:31-2.

12. Isaia GC, Casali S, Grosso I, Molinatti PA, Tamone C, Sategna-Guidetti C. Hypoparathyroidism and co-existing celiac disease. J Endocrinol Invest. 2004;27:778-81.

13. Khandwala HM, Chibbar R, Bedi A. Celiac disease occurring in a patient with hypoparathyroidism and autoimmune thyroid disease. South Med J. 2006;99:290-2.

14. Gelfand IM, Dimeglio LA. Hypocalcemia as a presenting feature of celiac disease in a patient with DiGeorge syndrome. J Pediatr Endocrinol Metab. 2007; 20:253-5. 